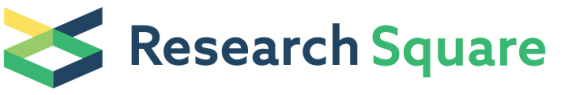 \\ Preprints are preliminary reports that have not undergone peer review. \\ They should not be considered conclusive, used to inform clinical practice, \\ or referenced by the media as validated information.
}

\section{The complexity of genomic mutation dictates the prognosis of acute leukemia with ambiguous lineage}

\author{
Jin Huang \\ Huazhong University of Science and Technology \\ Min Xiao \\ Huazhong University of Science and Technology \\ Jing Zhou \\ Huazhong University of Science and Technology \\ Xia Mao \\ Huazhong University of Science and Technology \\ Li Zhu \\ Huazhong University of Science and Technology \\ Songya Liu \\ Huazhong University of Science and Technology \\ Qinlu Li \\ Huazhong University of Science and Technology \\ Jin Wang \\ Huazhong University of Science and Technology \\ Jianfeng Zhou \\ Huazhong University of Science and Technology \\ Haodong Cai \\ Huazhong University of Science and Technology \\ Gaoxiang Wang ( gxwtjxy@126.com) \\ Huazhong University of Science and Technology
}

\section{Research Article}

Keywords: acute leukemia with ambiguous lineage, gene mutation, next-generation sequencing, prognosis

Posted Date: March 29th, 2021

DOI: https://doi.org/10.21203/rs.3.rs-108785/v2

License: (c) (i) This work is licensed under a Creative Commons Attribution 4.0 International License. Read Full License 


\section{Abstract}

Acute leukemia with ambiguous lineage (ALAL) is a rare and highly aggressive malignancy with limited molecular characterization and therapeutic recommendations. In this study, we retrospectively analyzed 1635 acute leukemia cases in our center from January 2012 to June 2018. The diagnose of ALAL was based on either EGIL or 2016 WHO criteria, a total of 39 patients were included. Four patients diagnosed as acute undifferentiated leukemia (AUL) by both classification systems. The mutations detected in bi-phenotypic acute leukemia enriched in genes related to genomic stability and transcriptional regulation; while AUL cases frequently mutated in genes involved in signaling pathway. Survival analysis of all patients suggested that the prognosis of ALAL was independent of immunophenotype, chromosome karyotype, treatment, but significantly associated with the mutation complexity, also termed numbers of the mutations carried by each patient (Log rank $p=0.009$ for progression-free survival [PFS] and Log rank $p=0.047$ for overall survival [OS], respectively). Similar results were obtained when the WHO diagnostic system were applied (Log rank $p<0.001$ for both PFS and OS). Among these patients, those excluded by WHO criteria had even worse clinical outcome than the patients included (Log rank $p=0.023$ for PFS and Log rank $p=0.031$ for OS). Collectively, the complexity of genomic mutation of ALAL patients is significantly associated with the clinical outcomes. The rationality and clinical applicability of the diagnostic criteria of WHO system need to be evaluated by more largescale clinical studies.

\section{Introduction}

Majority of acute leukemia (AL) can be accurately diagnosed and classified into acute myeloid leukemia (AML) or acute lymphoid leukemia (ALL) according to morphologic, cytochemical, and immunologic features ${ }^{1}$. However, less than $5 \%$ of patients can't be clearly assigned to the well-established subsets of AL as lacking expression of lineage-specific markers, or presenting mixed immune phenotype more than one lineage. In the past two decades, the diagnostic criteria for such kinds of leukemia were controversial, and the nomenclature was constantly improving. The European Group for the immunological characterization of leukemia (EGIL) separately defines them as acute undifferentiated leukemia (AUL), which means no lineage related antigens are expressed, and bi-phenotypic acute leukemia (BAL) when either two separate blast populations are encountered or a single blast population demonstrating evidence of both myeloid and lymphoid differentiation concurrently ${ }^{2}$. WHO (2008/2016) classification system collectively groups these rare disorders as acute leukemia with ambiguous lineage (ALAL), which may possibly arise from hematopoietic pluripotent stem cells ${ }^{3}$. The new classification system proposes the diagnosis of mixed-phenotype acute leukemia (MPAL) and emphasizes the impact of CD19 for B-cell lineage, CD3/cCD3 for Tcell lineage, and cytoplasmatic myeloperoxidase (MPO) for myeloid lineage, respectively ${ }^{4,5}$. The diagnosis of ALAL largely depends on the immunophenotype, and cases with AML specific recurrent cytogenetic abnormalities are classified to AML as defined in WHO classification system. Based on the markers expressed on the blast cells, BAL/MPAL can be further divided in to $\mathrm{B} / \mathrm{M}, \mathrm{T} / \mathrm{M}, \mathrm{B} / \mathrm{T}$, and $\mathrm{B} / \mathrm{T} / \mathrm{M}$ subtypes.

It has been widely recognized that the clinical outcome of ALAL is markedly adverse, and the overall survival (OS) ranges from 9 months to 3.5 years according to published data ${ }^{6-8}$. Poor response to conventional chemotherapy and high rate of relapse may partly explain the unsatisfactory clinical outcome. Some studies reported ALL-like therapy might be superior to AML-based protocols, and long-term survival can only be improved by allogeneic stem cell transplantation ${ }^{8-11}$. However, till now, no consistent treatment strategies are recommended and long-term survival in adult patients is less than $20 \%{ }^{10}$. Another important issue which can't be ignored is that the new WHO diagnostic system, compared with the previous EGIL criteria, eliminates a certain body of patients from ALAL. These patients may be diagnosed as AML or ALL, and receive the chemotherapy accordingly. Whether their diagnosis and treatments are appropriate needs more comprehensively evaluation.

Better characterizing this subset of leukemia will shed new light in overcoming this special entity of disease. However, no distinctive biological characteristics have been identified. As previous studies documented, cytogenetic abnormalities were detected in $59-91 \%$ of patients with MPAL and $80-90 \%$ of patients with AUL. However, no single abnormalities were unique to ALAL except for rearrangement of ZNF384 commonly appeared in B/M MPAL ${ }^{12}$. In recent years, progressions in molecular genetics and high-throughput sequencing technology make it possible to investigate this kind of disease more profoundly in 
molecular level 6,13,14. Several studies demonstrated the genetic landscape of MPAL and showed patients clustered to either AML-like or ALL-like MPAL based on methylation profiling could be benefited from corresponding therapies ${ }^{13,14}$. However, the correlation between the genetic mutation and the clinical outcomes of ALAL was not fully elucidated.

In this study, we retrospectively analyzed the patients with de novo acute leukemia from January 2012 to June 2018 in Tongji Hospital, Tongji Medical Collage of Huazhong University of Science and Technology and aimed to examine a wide spectrum of gene mutations in patients with ALAL with defined under WHO or EGIL criteria, to determine their clinical relevance with clinical outcomes of such dire leukemia and the clinical applicability of these two diagnostic systems.

\section{Results}

\section{Basic characteristics of patients enrolled in this study}

In all 1635 patients with acute leukemia admitted to our center over a period of more than five years, 39 patients $(2.4 \%)$ satisfied the criteria of EGIL, and 30 (1.8\%) patients were diagnosed as AUL or MPAL according to the WHO standards. For all 39 patients, mean age was 39 years and $23(59 \%)$ of them were male. The median value of WBC count on presentation was $11 \times 10^{9}$, hemoglobin $80 \mathrm{~g} / \mathrm{L}$, platelet $71 \times 10^{9}$, and bone marrow blasts $80 \%$ (Table 1 ). There was no significant difference regarding to the baseline and clinical characteristics between the patients included (WHO 2016, n=30) and excluded (EGILWHO, $n=9)$ by WHO criteria, except that patients excluded by WHO system had a lower level of platelet count $(p=0.039)$ and did not express MPO ( $p=0.001)$. According to the FAB criteria, $14(35.9 \%)$ patients were classified as ALL, and $5(12.8 \%)$ patients displayed an AML morphology, and others were classified as AUL (7.7\%), mix phenotype leukemia (2.6\%), or inclusive (41\%) (Supplementary Table 1). The clinical diagnosis of ALAL can hardly be realized by FAB diagnosis system 
Clinical and laboratory features of patients in this study

\begin{tabular}{|c|c|c|c|c|}
\hline \multirow[t]{2}{*}{ Characteristics } & \multirow{2}{*}{$\begin{array}{l}\text { EGIL } \\
(n=39)\end{array}$} & \multirow{2}{*}{$\begin{array}{l}\text { WHO } 2016 \\
(n=30)\end{array}$} & \multirow{2}{*}{$\begin{array}{l}\text { EGIL-WHO } \\
(n=9)\end{array}$} & \multirow[t]{2}{*}{ p value } \\
\hline & & & & \\
\hline Sex, m/f & $23 / 16$ & $18 / 12$ & $5 / 4$ & 0.812 \\
\hline Age, y & $39 \pm 16.6$ & $39 \pm 14.9$ & $38 \pm 22.4$ & 0.935 \\
\hline WBC, $\times 10^{9} / \mathrm{L}$ & $11(3-40)$ & $10(3-39)$ & $37(2-107)$ & 0.588 \\
\hline$H B, g / L$ & $80(62-106)$ & $86(64-105)$ & $68(42-124)$ & 0.501 \\
\hline $\mathrm{PLT}, \times 10^{9} / \mathrm{L}$ & $71(35-131)$ & $79(44-145)$ & $29(7-80)$ & 0.039 \\
\hline BM blast (\%) & $80(55-92)$ & $76(51-90)$ & $92(75-97)$ & 0.069 \\
\hline \multicolumn{5}{|l|}{ Karyotype abnormality, N (\%) } \\
\hline Abnormal & $17(53.1)$ & $15(60.0)$ & $2(28.6)$ & 0.141 \\
\hline $\mathrm{Ph}+$ & 7 (21.9) & $7(28.0)$ & $0(0)$ & 0.113 \\
\hline MLL rearrangment & $1(3.1)$ & $0(0)$ & $1(14.3)$ & 0.055 \\
\hline Complex & $9(28.1)$ & $8(32.0)$ & $1(14.3)$ & 0.357 \\
\hline \multicolumn{5}{|l|}{ Immunophenotype profile, N/tested } \\
\hline CD34+ & $36 / 39$ & $27 / 30$ & $9 / 9$ & 0.323 \\
\hline MPO+ & $19 / 39$ & $19 / 30$ & $0 / 9$ & 0.001 \\
\hline CD13+ & $30 / 38$ & $22 / 29$ & $8 / 9$ & 0.402 \\
\hline CD11c+ & $9 / 17$ & $7 / 13$ & $2 / 4$ & 0.893 \\
\hline CD14+ & $5 / 29$ & $3 / 21$ & $2 / 8$ & 0.495 \\
\hline CD64 & $6 / 25$ & $5 / 17$ & $1 / 8$ & 0.356 \\
\hline CD33+ & $24 / 34$ & $17 / 25$ & $7 / 9$ & 0.581 \\
\hline CD117+ & $24 / 36$ & $16 / 27$ & $8 / 9$ & 0.102 \\
\hline CD19+ & $17 / 38$ & $15 / 29$ & $2 / 9$ & 0.120 \\
\hline CD20+ & $4 / 23$ & $4 / 19$ & $0 / 4$ & 0.313 \\
\hline CD22+ & $10 / 13$ & $8 / 10$ & $2 / 3$ & 0.631 \\
\hline CD10+ & $18 / 32$ & $15 / 24$ & $3 / 8$ & 0.217 \\
\hline CD79a+ & $17 / 36$ & $14 / 27$ & $3 / 9$ & 0.335 \\
\hline CD3+/cCD3+ & $20 / 37$ & $14 / 28$ & $6 / 9$ & 0.383 \\
\hline CD2+ & $9 / 23$ & $6 / 16$ & $3 / 7$ & 0.809 \\
\hline CD5+ & $11 / 24$ & $9 / 16$ & $2 / 8$ & 0.148 \\
\hline CD7+ & $25 / 38$ & $18 / 29$ & $7 / 9$ & 0.386 \\
\hline Patients with mutations, N/tested & $17 / 19$ & $15 / 17$ & $2 / 2$ & 0.608 \\
\hline
\end{tabular}




\begin{tabular}{|llll|}
\hline Characteristics & EGIL & WHO 2016 & EGIL-WHO \\
\cline { 2 - 4 } & $(\mathbf{n = 3 9 )}$ & $(\mathbf{n = 3 0 )}$ & $(\mathbf{n = 9 )}$ \\
\hline p values value
\end{tabular}

Four AUL cases (10\%) expressed no lineage specific markers but CD34, HLA-DR with or without TdT. Among the remaining cases, $16(41 \%)$ cases had a myeloid and B-lymphoid phenotype (B-M), $16(41 \%)$ for a myeloid and T-lymphoid phenotype (T-M), $2(5.1 \%)$ for a B-T-lymphoid phenotype (B-T), and 1 cases (2.6\%) with evidence of tri-lineage concomitant expression (myeloid, $\mathrm{B}$, and T lymphoid [B-T-M]; Supplementary Table 1).

Among all, 32 patients underwent karyotyping, and 17 (53.1\%) of them were abnormal. Complex karyotypes were observed in 9 $(28.1 \%)$ patients with a high degree of heterogeneity as shown in Table 2 . The most frequent abnormal karyotype is $t(9 ; 22)$ (q34; q11.2), resulting in the BCR-ABL1 fusion gene, appeared in 7 patients. Interestingly, 6 patients were B-M MPAL while one of them was T-M MPAL. MLL rearrangement was observed in a patients diagnosed as T-M MPAL (Table 2). In addition to BCR-ABL and SET/CAN, which was previously reported, we also firstly detected E2A-PBX1 fusion genes in a B-M MPAL patient (Table 2). 
Table 2

Detailed information of the patients with cytogenetic abnormalities

\begin{tabular}{|c|c|c|c|c|c|c|c|c|}
\hline \multirow{2}{*}{$\begin{array}{l}\text { Patient } \\
\text { No. }\end{array}$} & \multirow[t]{2}{*}{ Gender } & \multirow{2}{*}{$\begin{array}{l}\text { Age } \\
\text { year }\end{array}$} & \multirow{2}{*}{$\begin{array}{l}\text { EGIL } \\
\text { classification }\end{array}$} & \multirow{2}{*}{$\begin{array}{l}2016 \text { WHO } \\
\text { classification }\end{array}$} & \multirow{2}{*}{$\begin{array}{l}\text { Gene } \\
\text { fusion }\end{array}$} & \multirow[t]{2}{*}{ Chromosome karyotype } & \multirow[t]{2}{*}{ CR } & \multirow{2}{*}{$\begin{array}{l}\text { OS } \\
\text { (day) }\end{array}$} \\
\hline & & & & & & & & \\
\hline 1 & Male & 24 & BAL & MPAL, B-M, NOS & $\mathrm{E} 2 \mathrm{~A} / \mathrm{PBX} 1$ & $\begin{array}{l}46, X Y,[9] / 43 \varangle X Y, b(1 q), 6 q- \\
-9, \\
-10,11 q-,-15,-17,-18,19 p-,-21 \\
{[1]}\end{array}$ & Yes & $478+$ \\
\hline 2 & Female & 42 & BAL & $\begin{array}{l}\text { MPAL, } \\
\text { BCR/ABL+ }\end{array}$ & $\begin{array}{l}\text { BCR/ABL } \\
(\mathrm{P} 210)\end{array}$ & $\begin{array}{l}46, X X, t(9 ; 22)(q 34 ; q 11) \\
{[20]}\end{array}$ & Yes & $115+$ \\
\hline 3 & Female & 24 & BAL & MPAL, B-M, NOS & Neg & $\begin{array}{l}\text { 46, XX, t (2:5) (p21:p15), } \\
\text { del (6) (q16-q22), t (9:x) } \\
\text { (p24:q21) }\end{array}$ & Yes & 578 \\
\hline 13 & Female & 56 & BAL & MPAL, T-B, NOS & Neg & $\begin{array}{l}\text { 46, XY, dic(11:22) } \\
\text { (q24; } 12) \text {,del(12)(q22) } \\
\text { [7]/46,xx[3] }\end{array}$ & No & 381 \\
\hline 15 & Male & 20 & BAL & MPAL,BCR/ABL+ & $\begin{array}{l}\mathrm{BCR} / \mathrm{ABL} \\
(\mathrm{P} 190)\end{array}$ & $\begin{array}{l}\text { 46,XY, r (5) (p15q35), t (9; } \\
\text { 22) (q34, q11) [14] / 46, } \\
\mathrm{XY}, \mathrm{t}(9 ; 22)(\mathrm{q} 34 ; \mathrm{q} 11) \text { [6] }\end{array}$ & Yes & $530+$ \\
\hline 16 & Female & 58 & BAL & MPAL, T-M, NOS & SET/CAN & Neg & No & 360 \\
\hline 19 & Male & 26 & BAL & MPAL, T-B, NOS & Neg & Subtriploid karyotype & Yes & $602+$ \\
\hline 21 & Female & 29 & BAL & MPAL,BCR/ABL+ & $\mathrm{BCR} / \mathrm{ABL}$ & $\begin{array}{l}46, X X[6] /[C P] 46, X X \\
2 q+, t(9 ; 22)(q 34 ; q 11) \\
14 p+[8]\end{array}$ & Yes & $579+$ \\
\hline 22 & Male & 25 & BAL & T-ALL & / & $\begin{array}{l}\text { 43-47, XY, t (4; 11) (q21; } \\
\text { p15), + 6, del (6) (q21), del } \\
\text { (7), (q21), del (7) (q14), } \\
\text { add (9) (p24), add (11) } \\
\text { (p15), add (14) (q32),-17, } \\
\text { del (17), (p13), +ace [cp6] / } \\
46, X Y,[14]\end{array}$ & No & 83 \\
\hline 25 & Female & 43 & BAL & MPAL, T-M, NOS & / & $47, X X,+21[4] / 46, X X[2]$ & Yes & $545+$ \\
\hline 26 & Female & 41 & BAL & MPAL,BCR/ABL+ & $\begin{array}{l}\text { BCR/ABL } \\
(\mathrm{P} 210)\end{array}$ & / & Yes & $2293+$ \\
\hline 29 & Female & 60 & BAL & B-ALL & Neg & $\begin{array}{l}47, \mathrm{XX}, \mathrm{t}(8 ; 13),(q 10 ; \mathrm{p} 10) \\
+22[10]\end{array}$ & No & 51 \\
\hline 31 & Male & 50 & BAL & MPAL,BCR/ABL+ & $\begin{array}{l}\text { BCR/ABL } \\
(\mathrm{P} 210)\end{array}$ & $47, X X,+11[9] / 46, X X,[1]$ & Yes & $850+$ \\
\hline 32 & Female & 36 & AUL & AUL & Neg & $47, x X,+8[3]$ & No & 382 \\
\hline 36 & Male & 18 & BAL & MPAL,BCR/ABL+ & $\begin{array}{l}\text { BCR/ABL } \\
(\mathrm{P} 190)\end{array}$ & Neg & Yes & $565+$ \\
\hline 37 & Male & 42 & BAL & MPAL,BCR/ABL+ & $\begin{array}{l}\text { BCR/ABL } \\
(\mathrm{P} 190)\end{array}$ & $\begin{array}{l}\text { 49, XY, t (9; 22) (q34; q11), } \\
\text { + 10, +21, +der (22) t (9; } \\
\text { 22) (q34; q11) [1]/ 53, } \\
\text { idem, M31 + X, + 1, +5, + } 8 \\
\text { [1] / 46, xy [18] }\end{array}$ & No & 731 \\
\hline 44 & Male & 44 & BAL & MPAL, T-M, NOS & Neg & $46, X Y[6] / 47, X Y,+10[14]$ & Yes & $351+$ \\
\hline 45 & Male & 23 & AUL & AUL & Neg & $\begin{array}{l}47, \mathrm{XY} \text {, add (2) (q37), + 4, t } \\
(10 ; 11) \text { (p12;q21), -17, } \\
\text { +mar [4]/47, idem, add (2) } \\
\text { (q37) [1] /46, XY, [15] }\end{array}$ & Yes & 141 \\
\hline
\end{tabular}




\section{Genetic testing}

Genetic testing was conducted in 27 cases. Nineteen out of them were tested by 173-gene panel (Supplementary Table 2) in next-generation deep sequencing platform, and 8 of them, who admitted in hospital in earlier time, were tested by Sanger sequencing. 17 cases (89.5\%) were detected at least one somatic mutation by high-throughput sequencing system, while only 2 patients were positive mutated by screening limited hot spot mutation of AML by Sanger sequencing. The two mutated genes detected by first-generation sequencing were the CEBPA in No.36 patient and the FLT3-ITD repetitive tandem sequence in No. 42 patient, respectively.

Based on the data of the 19 patients who had been sequenced in the next-generation platform, 53 high-confidence somatic mutations were detected in 33 genes in 17 patients. The two patients with no detectable mutations were both B-M phenotype. The median number of gene mutation was $3(0-8)$ per sample. The most frequently mutated genes were NRAS (4, 21\%), CEBPA (4, 21\%), JAK3 (3, 16\%), RUNX1 (3, 16\%). Mutations in genes such as DNMT3A, ETV6, IDH2, KMT2D, KRAS, NOTCH1, PHF6, TP53, and WT1 were detected in two different patients. CEBPA mutations were detected in 3 patients with M-T phenotype and one with B-T phenotype. Notably, the mutations in NRAS gene (reoccurring in 4 cases) were concentrated at its 12th/13th amino acid site.

We divided genes into the following categories according to gene attribute: transcription factor, chromatin regulation, epigenetics, cell apoptosis, signaling pathway (RTK-RAS, NOTCH, MAPK-ERK, PI3K/AKT, JAK-STAT), and others. The results showed that mutations detected in BAL cases enriched in transcription factor, chromatin regulation and epigenetic, all related to genomic stability and transcriptional regulation; while AUL cases frequently mutated in genes in signal pathway regulation such as RTK-RAS, NOTCH, and PI3K/AKT (Fig. 1A). There was no significant difference in the distribution of B/M and T/M gene mutations, except for the particularly high frequency of transcription factor mutations in T/M cases (71.4\%) (Fig. 1B). The detailed information profile was shown in Fig. 1C.

\section{Survival Analyses}

Survival analyses were used to compare the prognosis of clinical subsets and types of treatment. Up to January 16, 2019 (mean follow up time 428 days), 34 patients received chemotherapy, while others, according to patients' wishes or limitation of general condition, only received symptomatic support treatment. Among the patients who received chemotherapy, 7 cases received ALL-like therapy, 6 cases received AML-like therapy, and 21 cases received AML+ALL combined therapy. Decitabine was included in the therapy of 9 patients, 5 patients received tyrosine kinase inhibitors (TKI), and 4 patients received allogeneic hematopoietic stem cell transplantation (HSCT).

Among all the patients, 21 patients (53.8\%) achieved complete remission (CR) at least once. The CR rate was not affected by immunophenotype $(p=0.167)$, complex karyotype $(p=0.761)$, whether mutated $(p=0.156)$, or chemotherapy regimen $(p=0.326)$; while showed a slight association with no. of mutation/person $(p=0.096)$ (Supplementary Table 3). During the follow up, 27 cases (69.2\%) progressed or died. Of all factors considered, mutation complexity, defined as no. of mutation/person was significantly associated with PFS and OS (Log rank $p=0.009$ and Log rank $p=0.047$ respectively) (Table 3 and Fig. 2A-B). When the WHO diagnostic criteria were applied, we obtained the similar results in the 30 patients included. In specific, mutation complexity was the only clinical risk factor that remarkably associated with PFS (Log rank $p<0.001$ ), and OS (Log rank $\mathrm{p}<0.001$ ), manifested as patients with more complex mutation profiles had much worse clinical outcomes (Supplementary Table 3 and Fig. 2C-D). Of note, 9 patients were excluded according to WHO 2016 classification system. Eight of them were diagnosed as acute lymphoblastic leukemia, and one was determined as unclassified. We found the patients excluded by WHO criteria had an even worse prognosis than those patients included, characterized as shorter PFS (Log rank $p=0.023)$ and OS (Log rank $\mathrm{p}=0.031$ ) (Table 3 and Fig. 2E-F). Whether the clinical diagnosis and treatment of these subsets of patients is the optimal strategy may need to be further explored.

\section{Discussion}


ALAL is a rare and aggressive entity with heterogeneous immunophenotypic, cytogenetic and molecular features. Our study provided the genetic landscape of ALAL by screening the hot-spots in 173 genes strongly associated myeloid and lymphoid malignancies and evaluated the clinical characteristics with the dismal clinical outcomes. Apart from immunophenotype, complex karyotype, treatment, somatic mutation complexity is exclusively associated PFS and OS in ALAL. The increased number of mutations in blast cells predicted higher risk of relapses and deaths, no matter using the WHO or the EGIL diagnostic system to evaluate. Recent studies also emphasized the important roles of somatic mutations in the diagnoses and treatment strategy of ALAL,but most of published studies focused on MPAL $6,13,14$. In this study, we firstly analyzed 4 patients with AUL by high throughput sequencing and demonstrated these two sub-types of ALAL possessed different mutation files. We anticipate that the genetic information revealed by high throughput sequencing will innovate the medical care of ALAL in the future.

Another important point of view of our study is the deep exploration of the diagnostic system of ALAL. EGIL classification guideline was first proposed in 1995 and adopted in WHO criteria in 20012. Updated WHO 2008/2016 classification implicated significant modification to the EGIL score system and quite a number of patients were eliminated outside ALAL diagnosis, just as we found in our study 4,5 . This part of patients was diagnosed as AML or ALL, and received corresponding treatment. However, whether the remarkable change is suitable and beneficial for the patients is still controversial, and the conflict focuses on the part of patients excluded by the new WHO system ${ }^{3}$. In this study, we found the patients excluded by WHO system had an even worse prognosis (Log rank $p=0.023$ for PFS and Log rank $p=0.031$ for OS), which was consistent with a previous reported clinical study ${ }^{15}$. In spite of this, we also found that the patients, satisfied the EGIL criteria but excluded by WHO diagnose system, shared the similar basic characteristics and immunophenotype with the patients included as showed in Table 1. Of the 9 patients excluded by WHO from ALAL, 2 cases underwent high throughput sequencing, and the results showed both of them carried completed genetic mutations distributed in genes like PTEN, KRAS, JAK3, KMT2C, WT1, ETV6, TP53, JAK3, RUX1, NOTCH, PHF6, and CREBBP. One of them showed no response to conventional chemotherapy, and unfortunately died 83 days after the diagnose. This is the first study to investigate the genetic background of this part of patients, however, the number of patients is limited to describe the whole picture of these patients. As the diagnosis significantly dictates the therapeutic decision making, the clinical appliance of the WHO 2008/2016 criteria needs to be in more prospective clinical studies.

The role of genetic (cytogenetic and molecular) markers in guiding chemotherapy strategies as well as targeted therapy is inexperienced. According to the WHO2016 criteria, $\mathrm{t}(9 ; 22) / \mathrm{Ph}+$ was considered as a separate entity. And the Ph chromosome was revealed as the most common cytogenetic abnormality, with the incidence ranging from $17-41 \%$ in MPAL especially with B-M phenotype ${ }^{10}$. All $\mathrm{Ph}+$ patients should be confirmed as soon as possible due to the benefit probability from $\mathrm{TKI}^{16,17}$. In our study, we not only screened out 7 abnormal karyotyping patients with $P h+$ resulting in $B C R / A B L$ fusion gene, but also further detected 1 case with T315I mutation. Therefore, it is evident for us to make a better choice on the utility of TKIs. New molecular information in combination with cytogenetic characteristics will be valuable for the identification of genetic changes in ALAL, and undoubtedly play an important role in precision medicine of ALAL.

IDH2 gene mutations frequently include R140Q and R172K ${ }^{18}$. Surprisingly among our 19 available samples for NGS, we found 2 cases (AUL: $n=1$, MPAL, T/M, NOS: $n=1$ ) harboring IDH2 R140Q mutation. Recently, novel selective inhibitors of IDH1/2 mutations, ivosidenib and enasidenib, have been approved for patients with relapsed or refractory IDH1/2-mutated AML ${ }^{18}$. There is a proof that a patient with IDH1-mutated AUL achieved molecular complete remission following ivosidenib ${ }^{19}$. This might be a promising step to open the door for targeted therapy in IDH-mutated $\operatorname{ALAL}^{20}$.

Interestingly, we first found E2A/PBX1 in MPAL, B/M, NOS, which has not been reported in ALAL before. To our knowledge, it is one of the most common translocations in pediatric B-ALL. In adult, although not exceptional, it appears approximately $3 \% 20$. E2A/PBX1 fusion gene is associated with unfavorable prognosis, eventually leading to the intensive therapeutic approaches. The prognostic significance of E2A/PBX1 fusion gene in ALAL is acquired further research.

Given that the drugable genetic target is still limited, analyzing the aberrant or specific antigen expression on leukemia cells seems to be another potential strategy. We tried to detect some canonical surface markers which can be targeted by off-the- 
shelf chimeric antigen receptor T-cell (CAR-T) immunotherapy. Strikingly, we definitely found 11 patients (47.8\% of tested, data not shown) expressed CD123 on the surface of the blast cells. It has been reported that CD123, as a more specific marker for AML cells, is expressed at low levels on normal hematopoietic stem/progenitor cells ${ }^{21}$. And CD123 CAR-T therapy exhibited a good effect in patients who are no longer responding to standard therapies ${ }^{22,23}$. It is potentially considered as a novel magic bullet for the therapeutics of ALAL with CD123 expression.

Collectively, as the ALAL appears heterogeneous, it seems reasonable to establish stratification system of diagnosis and prognosis, especially based on the genetic features. Elucidating the genetic heterogeneity implicated in the process of leukemogenesis will provide insights into the pathogenesis and improve the management of the unusual subtype of acute leukemia.

\section{Methods}

\section{Study Design and Participants}

A retrospective analysis was performed based on data from 1635 de novo acute leukemia patients in Tongji Hospital (Wuhan, China) from January 2012 to June 2018. Standard procedures for diagnosis were performed, including morphology examination, multi-parameter flow cytometry analysis, molecular detection and karyotype analysis. According to EGIL or 2016 WHO criteria, 39 (2.4\%) patients were enrolled in this study ${ }^{1,4,5}$. A total of 35 patients were designated as BAL according to EGIL scoring system and/or as MPAL if 2016 WHO criteria were fulfilled and 4 patients were diagnosed as AUL. The clinical characteristics, treatments and prognostic information were objectively collected from the medical records and validated by the physicians in charge or the telephone follow-up to the patients. ALL-like therapy was defined as regimens including vincristine, doxorubicin, cyclophosphamide, L-asparaginase, methotrexate and steroids, and AML-like therapy was defined as initial chemotherapy including anthracyline, cytarabine, with or without hypomethylating (demethylating) agents.

The study design was approved by the Ethics Committee of Tongji Hospital, Tongji Medical College, Huazhong University of Science and Technology according to the Declaration of Helsinki and all patients gave written informed consents to participate in this study.

\section{Targeted gene next-generation sequencing and mutation calling}

Among the 19 patients underwent second-generation sequencing, 3 patients carried next-generation sequencing reports when they were admitted to our hospital, the data of next-generation sequencing was obtained from their medical record. For the others, samples from 15 patients were sequenced in lon Personal Genome Machine and 1 was sequences in Illumina NextSeq $500 / 550$ platform in our center. The panel designed for variants detection covered the main mutation hotspots of a total of 173 genes which were the most frequently detected in myeloid and lymphoid malignancies (Supplementary Table 2). Genomic DNA was isolated from tumor blasts from bone marrow samples with the QIAamp DNA Blood Kit (Qiagen GmbH, Hilden, Germany). Quality verified DNA extracted from tumor cells of patients was diluted to $5 \mathrm{ng} / \mu \mathrm{L}$ and prepared for library construction with the Ion AmpliSeq ${ }^{\text {TM }}$ Library Kit 2.0 (Applied Biosystems, Foster City, CA, USA). The concentration of library was measured by Qubit 3.0. All consumable items and reagents used in Library pooling, Emulsion PCR and Enrichment of template were provided in lon $\mathrm{PGM}^{\mathrm{TM}} \mathrm{Hi}-\mathrm{Q}^{\mathrm{TM}}$ View OT2 Kit and used according to the manufacturer's instructions. The next-generation sequencing was conducted in the lon torrent PGM (Applied Biosystems, Foster City, CA, USA) with lon 318 chip. The procedure of transferring raw signal to base and reads alignment were conducted in the local server by default settings. The VCF (Variant Call Format) files containing the raw mutation information was uploaded to lon Reporter server for further analysis. After getting MAF (Mutation Annotation Format) files, we filtered mutations by conditions: 1) MAF (Minor Allele Frequency) value $<0.01 ; 2$ ) Mutation reads number $>10$; 3) Coding region mutation; 4) Nonsynonymous mutation; 5) Not recorded in our false positive mutation database and human genome database of 1000 Genomes. IGV24 software was used to screening all mutations in BAM to guarantee no false positive mutations to be left ${ }^{25}$. 


\section{Statistical Analysis:}

The baseline characteristics were compared by independent sample $t$ test, chi square test, or non-parameter test when appropriate. OS was measured from the date of diagnosis to the date of last follow-up or death from any cause. For progression-free survival (PFS) calculation, the uncensored event was relapse of leukemia and the censoring times reflected the end of follow up without an event or lost to follow up. The Kaplan-Meier survival analysis method was used to evaluate the prognosis determinants. The estimated survival values in different subsets were compared using the log-rank test and $p<0.05$ were considered statistically significant. All analyses were performed using SPSS 21.0 (SPSS, Chicago, IL, USA).

\section{Declarations}

\section{Acknowledgement}

This research was funded by the grants from National Science Foundation of China (Young Scientist Fund, No. 82000176, 82000189 and No. 81800356), We thank all the study researchers, as well as patients who participated in the present study.

\section{Authors' contributions}

Haodong Cai and Gaoxiang Wang were responsible for the design and overall coordination of the study, and manuscript revising. Jin Huang is responsible for data analyzing and manuscript writing. Min Xiao is responsible for the genetic testing and sequencing data interpretation. Jing Zhou is responsible for the follow-up, and the other authors took part in the clinical testing and data collection.

\section{Additional information}

\section{Ethics approval and consent to participate}

The study design was approved by the Ethics Committee of Tongji Hospital, Tongji Medical College, Huazhong University of Science and Technology according to the Declaration of Helsinki and all patients gave written informed consents to participate in this study.

\section{Consent for publication}

All authors are aware of and agree to this submission.

\section{Availability of data and materials}

The raw data of next-generation sequencing are available at SRA (the sequence read archive) database of NCBI website (Bioproject: PRJNA646819).

\section{Competing interests}

The authors have no conflict of interest.

\section{Abbreviations}

$\mathrm{AL} \quad$ acute leukemia

ALAL acute leukemia with ambiguous lineage

ALL acute lymphoid leukemia

AML acute myeloid leukemia 
AUL acute undifferentiated leukemia

BAL bi-phenotypic acute leukemia

CAR-T chimeric antigen receptor T-cell

CR complete remission

EGIL European Group for the immunological characterization of leukemia

HSCT hematopoietic stem cell transplantation

MPAL mixed-phenotype acute leukemia

OS overall survival

PFS progression free survival

TKI tyrosine kinase inhibitors

\section{References}

1. Bene, M. C. et al. Proposals for the immunological classification of acute leukemias. European Group for the Immunological Characterization of Leukemias (EGIL). Leukemia. 9, 1783-1786 (1995).

2. van Dongen, J. J. Proposals for immunological classification of acute leukemias. Leukemia. 9, 2149-2150 (1995).

3. van den Ancker, W. et al. Acute leukemias of ambiguous lineage: diagnostic consequences of the WHO2008 classification. Leukemia. 24, 1392-1396 https://doi.org/10.1038/leu.2010.119 (2010).

4. Vardiman, J. W. et al. The 2008 revision of the World Health Organization (WHO) classification of myeloid neoplasms and acute leukemia: rationale and important changes. Blood. 114, 937-951 https://doi.org/10.1182/blood-2009-03-209262 (2009).

5. Arber, D. A. et al. The 2016 revision to the World Health Organization classification of myeloid neoplasms and acute leukemia. Blood. 127, 2391-2405 https://doi.org/10.1182/blood-2016-03-643544 (2016).

6. Yan, L. et al. Clinical, immunophenotypic, cytogenetic, and molecular genetic features in 117 adult patients with mixedphenotype acute leukemia defined by WHO-2008 classification. Haematologica. 97, 1708-1712 https://doi.org/10.3324/haematol.2012.064485 (2012).

7. Weinberg, O. K., Seetharam, M., Ren, L., Alizadeh, A. \& Arber, D. A. Mixed phenotype acute leukemia: A study of 61 cases using World Health Organization and European Group for the Immunological Classification of Leukaemias criteria. American journal of clinical pathology. 142, 803-808 https://doi.org/10.1309/AJCPPVUPOTUVOIB5 (2014).

8. Zheng, C. et al. What is the optimal treatment for biphenotypic acute leukemia? Haematologica. 94, 1778-1780 author reply 1780 https://doi.org/10.3324/haematol.2009.014829 (2009).

9. Heesch, S. et al. Acute leukemias of ambiguous lineage in adults: molecular and clinical characterization. Annals of hematology. 92, 747-758 https://doi.org/10.1007/s00277-013-1694-4 (2013).

10. Matutes, E. et al. Mixed-phenotype acute leukemia: clinical and laboratory features and outcome in 100 patients defined according to the WHO 2008 classification. Blood. 117, 3163-3171 https://doi.org/10.1182/blood-2010-10-314682 (2011).

11. Liu, Q. F. et al. Allo-HSCT for acute leukemia of ambiguous lineage in adults: the comparison between standard conditioning and intensified conditioning regimens. Annals of hematology. 92, 679-687 https://doi.org/10.1007/s00277012-1662-4 (2013).

12. Manola, K. N. Cytogenetic abnormalities in acute leukaemia of ambiguous lineage: an overview. British journal of haematology. 163, 24-39 https://doi.org/10.1111/bjh.12484 (2013). 
13. Takahashi, K. et al. Integrative genomic analysis of adult mixed phenotype acute leukemia delineates lineage associated molecular subtypes. Nature communications. 9, 2670 https://doi.org/10.1038/s41467-018-04924-z (2018).

14. Alexander, T. B. et al. The genetic basis and cell of origin of mixed phenotype acute leukaemia. Nature. 562, 373-379 https://doi.org/10.1038/s41586-018-0436-0 (2018).

15. Pomerantz, A. et al. Mixed-phenotype acute leukemia: suboptimal treatment when the $2008 / 2016$ WHO classification is used. Blood research. 51, 233-241 https://doi.org/10.5045/br.2016.51.4.233 (2016).

16. Wolach, O. \& Stone, R. M. How I treat mixed-phenotype acute leukemia. Blood. 125, 2477-2485 https://doi.org/10.1182/blood-2014-10-551465 (2015).

17. Roberts, K. G. et al. Targetable kinase-activating lesions in Ph-like acute lymphoblastic leukemia. The New England journal of medicine. 371, 1005-1015 https://doi.org/10.1056/NEJMoa1403088 (2014).

18. Dugan, J. \& Pollyea, D. Enasidenib for the treatment of acute myeloid leukemia. Expert review of clinical pharmacology. 11, 755-760 https://doi.org/10.1080/17512433.2018.1477585 (2018).

19. Patel, S. H. et al. Molecular Complete Remission Following Ivosidenib in a Patient With an Acute Undifferentiated Leukemia. Journal of the National Comprehensive Cancer Network: JNCCN. 18, 6-10 https://doi.org/10.6004/jnccn.2019.7368 (2020).

20. Foa, R. et al. E2A-PBX1 fusion in adult acute lymphoblastic leukaemia: biological and clinical features. British journal of haematology. 120, 484-487 https://doi.org/10.1046/j.1365-2141.2003.04113.x (2003).

21. Jordan, C. T. et al. The interleukin-3 receptor alpha chain is a unique marker for human acute myelogenous leukemia stem cells. Leukemia. 14, 1777-1784 https://doi.org/10.1038/sj.leu.2401903 (2000).

22. Mardiros, A. et al. T cells expressing CD123-specific chimeric antigen receptors exhibit specific cytolytic effector functions and antitumor effects against human acute myeloid leukemia. Blood. 122, 3138-3148 https://doi.org/10.1182/blood2012-12-474056 (2013).

23. Loff, S. et al. Rapidly Switchable Universal CAR-T Cells for Treatment of CD123-Positive Leukemia. Mol Ther Oncolytics. 17, 408-420 https://doi.org/10.1016/j.omto.2020.04.009 (2020).

24. Robinson, J. T. et al. Integrative genomics viewer. Nature biotechnology. 29, 24-26 https://doi.org/10.1038/nbt.1754 (2011).

25. McCall, C. M. et al. False positives in multiplex PCR-based next-generation sequencing have unique signatures. The Journal of molecular diagnostics: JMD. 16, 541-549 https://doi.org/10.1016/j.jmoldx.2014.06.001 (2014).

\section{Table 3}




\begin{tabular}{|c|c|c|c|c|}
\hline \multirow[t]{2}{*}{ Parameter } & \multirow{2}{*}{$\begin{array}{l}\text { PFS, day } \\
\text { median }\end{array}$} & \multirow{2}{*}{ p-value } & OS, day & \multirow[t]{2}{*}{ p-value ${ }^{\#}$} \\
\hline & & & median & \\
\hline \multicolumn{2}{|l|}{ Immunophenotype } & \multicolumn{2}{|l|}{0.184} & 0.217 \\
\hline AUL & \multicolumn{2}{|l|}{133} & \multicolumn{2}{|l|}{141} \\
\hline $\mathrm{B} / \mathrm{M}$ & \multicolumn{2}{|l|}{578} & \multicolumn{2}{|l|}{731} \\
\hline $\mathrm{T} / \mathrm{M}$ & \multicolumn{2}{|l|}{264} & \multicolumn{2}{|l|}{299} \\
\hline $\mathrm{B} / \mathrm{T}+\mathrm{B} / \mathrm{T} / \mathrm{M}$ & \multicolumn{2}{|l|}{205} & \multicolumn{2}{|l|}{205} \\
\hline \multicolumn{2}{|l|}{ Complex karyotype } & \multicolumn{2}{|l|}{0.936} & 0.741 \\
\hline Yes & \multicolumn{2}{|l|}{578} & \multicolumn{2}{|l|}{578} \\
\hline No & \multicolumn{2}{|l|}{338} & \multicolumn{2}{|l|}{360} \\
\hline \multicolumn{2}{|l|}{ Gene mutation } & \multicolumn{2}{|l|}{0.058} & 0.176 \\
\hline Yes & \multicolumn{2}{|l|}{252} & \multicolumn{2}{|l|}{382} \\
\hline No & \multicolumn{2}{|l|}{ / } & \multicolumn{2}{|l|}{ / } \\
\hline \multicolumn{2}{|l|}{ Mutation complexity\& } & \multicolumn{2}{|l|}{0.009} & 0.047 \\
\hline Low $(n=0 \square$ & / & & \multicolumn{2}{|l|}{ l } \\
\hline Middle $1 \leq \mathrm{n} \leq 3 \square$ & \multicolumn{2}{|l|}{338} & \multicolumn{2}{|l|}{731} \\
\hline High $\square n \geq 4 \square$ & \multicolumn{2}{|l|}{77} & 83 & \\
\hline Treatment & & 0.907 & & 0.145 \\
\hline ALL-like & 422 & & 478 & \\
\hline AML-like & 424 & & 1181 & \\
\hline AML+ALL-like & 338 & & 381 & \\
\hline Classification system & & 0.023 & & 0.031 \\
\hline WHO & 422 & & 578 & \\
\hline EGIL-WHO & 207 & & 211 & \\
\hline PFS: progression-free & : overall su & * evaluate & hi square & \\
\hline WHO: World Health Ors & EGIL: Europ & roup for th & sification & ute Leuken \\
\hline \# evaluated by Log-ran & ed as nun & pathoge & tations c & y each $p$ \\
\hline
\end{tabular}

Figures 
A

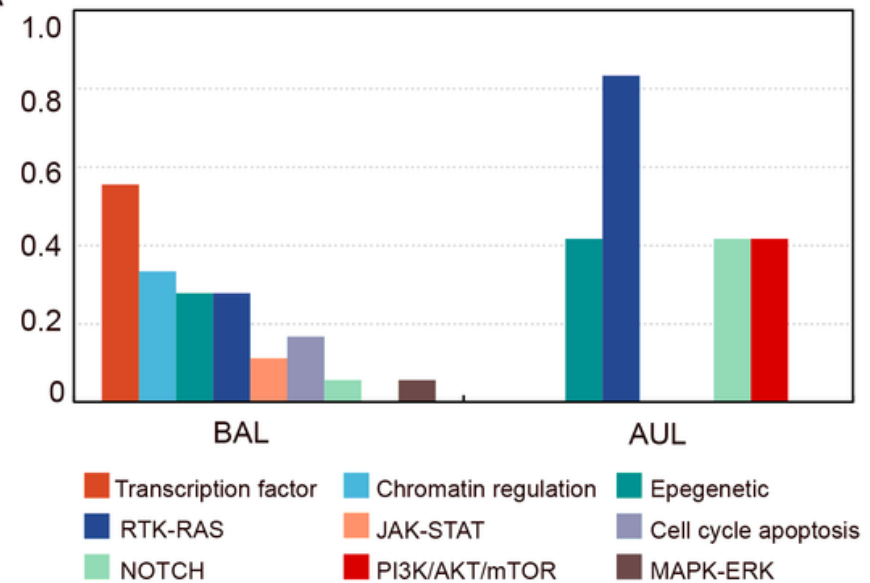

B

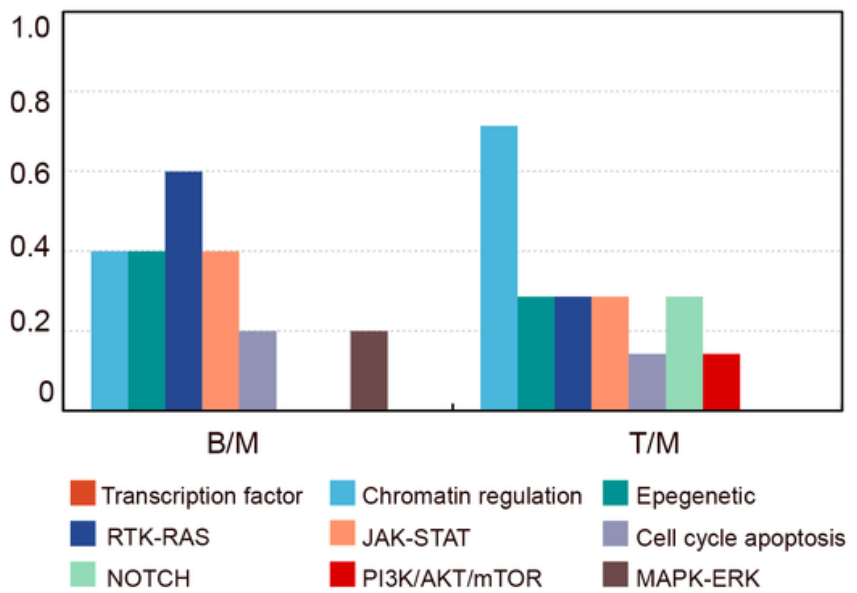

C

8
6
4
2
0
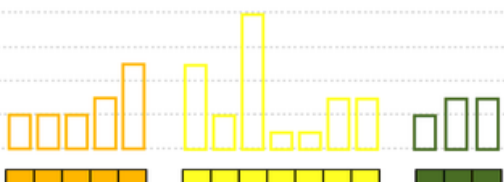

CEBPA
RUNX1
EVT6
IKZF1
PHF6
WT1
GATA2
DDX3X
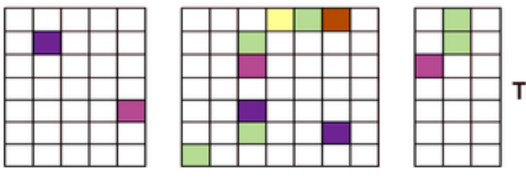

Transcription factor

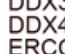

ERC

HIST $1 \mathrm{H}$

NPM1
RPL15

RPL15
SMC1A

KMT2D
CREBBP

CREBBP
DNMT3A

IDH2

KMT2C

KRAS

NRAS

FLT3
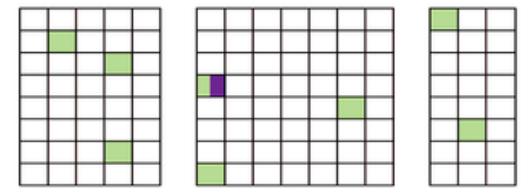

Chromatin regulation
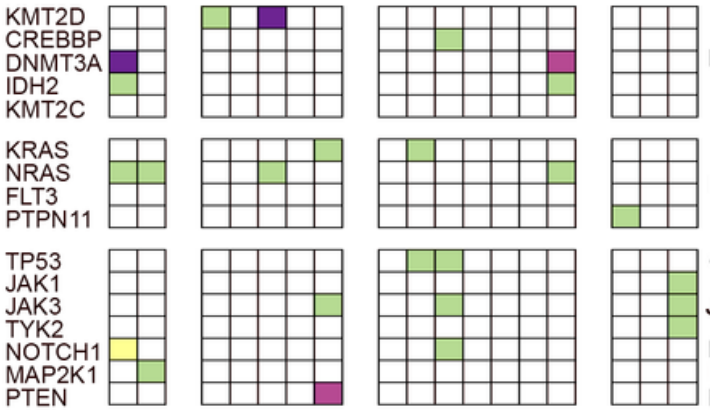

Epigenetic
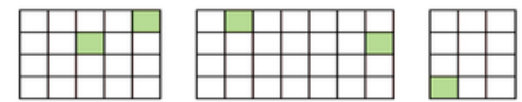

RTK-RAS
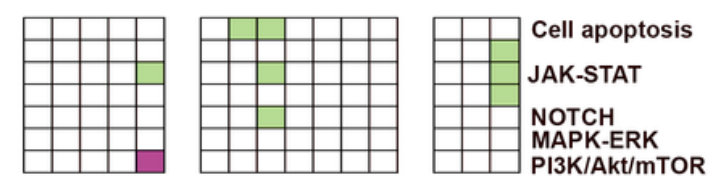

BCR/ABL
CD58
KIT

CCND3
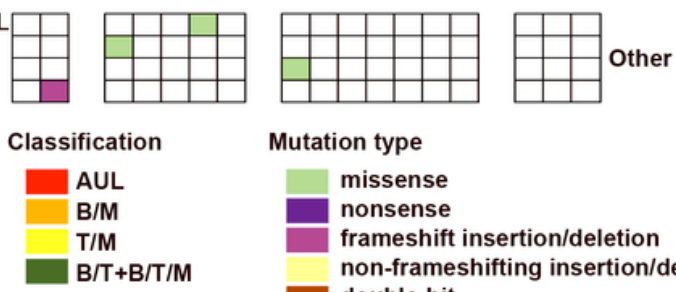

Mutation type

missense

nonsense

frameshift insertion/deletion

non-frameshifting insertion/deletion

double-hit

Figure 1

Landscape of somatic mutations in ALAL by next-generation sequencing. (A) Landscape of somatic mutations detected in biphenotypic acute leukemia (BAL, $n=15)$ and acute undifferentiated leukemia $(A U L, n=4)(B)$ Landscape of somatic mutations detected in myeloid/B-lymphoid phenotype $(B / M, n=5)$, and myeloid/T-lymphoid phenotype ( $T / M, n=7)$. Y-axis represents the ratio of patients with mutations and the patients performed next-generation genetic testing. Columns with different colors represent genes belong to different categories according their attributes. (C) Detailed information of the 17 cases who detected somatic mutations. Each column represents a case and each raw represents a gene. The top bar graph shows the number of mutations detected in each sample. The row directly underneath that graph show the immunophenotypes and Different colors of squares represent various mutation type. 

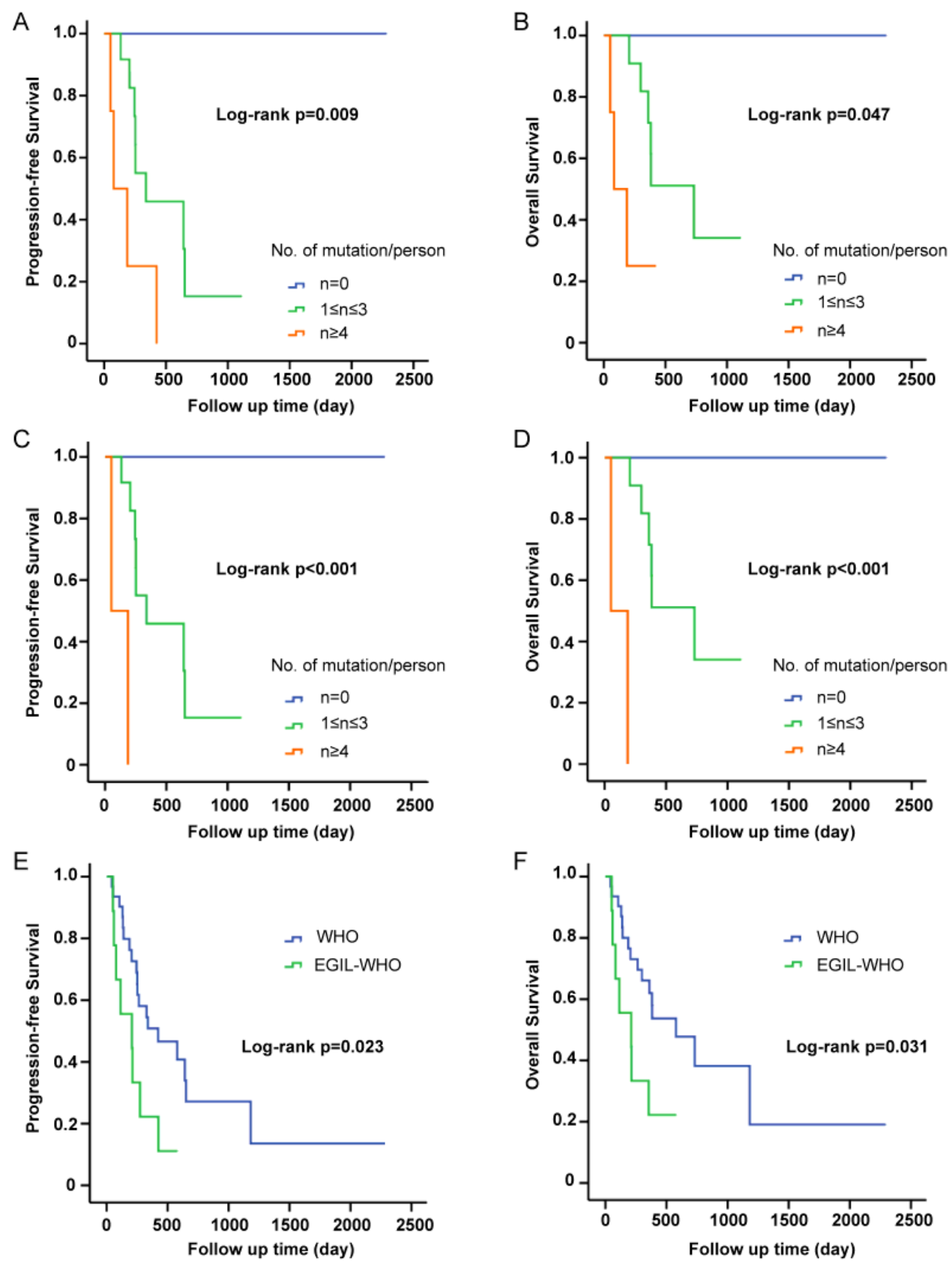

Figure 2

Outcomes of patients with ALAL by different clinical subsets. (A-B) Kaplan-Meier curve of PFS (A) and OS (B) stratified by no. of mutation/person by EGIL criteria. (C-D) Kaplan-Meier curve of PFS (C) and OS (D) stratified by no. of mutation/person by WHO 2016 criteria. (E-F) Kaplan-Meier curve of PFS (C) and OS (D) stratified by different classification systems (blue curves indicating patients included in WHO 2016 criteria and green curves indicating patients who were included by the EGIL classification but excluded by the WHO 2016 classification).

\section{Supplementary Files}

This is a list of supplementary files associated with this preprint. Click to download.

- SupplementaryMaterial.doc 